\title{
Arterial Stiffness and Pulse Wave Velocity in Healthy People of Older Age Groups
}

\author{
Amina Begum ${ }^{1}$, Shelina Begum ${ }^{2}$, Jahanara Arzu ${ }^{3}$, Md. Rezaul Karim Chowdhury ${ }^{4}$
}

\begin{abstract}
Background: Arterial stiffness is associated with endothelial dysfunction. Increased arterial stiffness well correlates not only with coronary risk factors but also with age. Objective: To evaluate the arterial stiffness by assessing pulse wave velocity (PWV) in apparently healthy people of different age groups. Methods: This cross sectional study was conducted on of 60 apparently healthy subjects with 19-49(younger age) and 50-75 (older age) years of age. To calculate PWV, Carotido-femoral length and Transit Time, was measured by a measuring tape and Doppler respectively. For statistical analysis, Independent Sample ' $t$ ' test and Pearson's correlation coefficient test was used. Results: In this study, PWV was significantly $(p<0.001)$ higher in older age group. PWV of younger age group in both male and female were within normal range. But in older age group, $20 \%$ had abnormal PWV. Moreover, PWV was positively correlated with age $\&$ it was significant $(\mathrm{p}<0.001)$. Conclusion: From this study, it may be concluded that arterial stiffness may develop in apparently healthy elderly subjects and they are more prone to develop hypertension.
\end{abstract}

Key words: Pulse wave velocity (PWV), Doppler method, Arterial stiffness.



\section{Introduction}

$\mathbf{C}$ ardiovascular diseases are globally recognized as one of the leading cause of death. Increased stiffness of the arterial wall is associated with the advancement of age worldwide ${ }^{1}$. Stiffness of arteries resulting from age or vascular pathology is attributed to $\operatorname{arterosclerosis}^{2}$. The pathogenesis of arteriosclerosis begins from thickening of its wall with luminal enlargement and terminates with loss of elastic properties in large arteries ${ }^{1}$. Arterial stiffening is one of the most commonly known risk factor, predisposed to advanced atherosclerotic vascular disease though, arterial stiffening can occur in the absence of

Received 14 Dec. 2017; $\quad$ Accepted 17 March 2018 atherosclerosis ${ }^{3}$. Advanced age related thickening of the arterial wall results from degeneration of elastin and increase in collagen fibers ${ }^{3}$.

Age related increase in the collagen concentration compared to elastin in all three layers of the arterial wall impaired the normal elastin: collagen balance that regulates arterial mechanics. Redistribution of collagen within tunica media is an usual feature over the age of 50 years $^{2}$.

However, among the different noninvasive methods used to assess arterial stiffness, determination of the carotid-femoral pulse wave 
velocity has been established as a gold standard due to its accuracy, reproducibility, relatively easy measurement and low costs ${ }^{-4}$. PWD (Pulse wave Doppler) echocardiography can be used easily and safely to measure arterial diseases ${ }^{5}$.

It is obvious that early diagnosis of vascular stiffness may be helpful for preventing various cardiovascular diseases as age increases. The routine screening of arterial stiffness may identify the risk population for hypertension ${ }^{6}$.Very few published data on PWV in older aged male and female healthy subjects are available. Therefore, the present study has been designed to observe the ageing effect on the distensibility of vascular wall by assessing PWV.

\section{Methods}

This cross sectional study was conducted in the Department of Physiology and Cardiology of Bangabandhu Sheikh Mujib Medical University (BSMMU), Dhaka, from March 2017 to February 2018. Protocol of this study was approved by the Institutional Review Board of BSMMU. For this study, 30 subjects aged 1949 years (younger age) and 30 subjects with 50 75 years (older age) of age were purposively selected. All subjects were apparently healthy and free from history of cardiovascular disease, diabetes mellitus (FPG $\geq 126 \mathrm{mg} / \mathrm{dl}(\geq 7 \mathrm{mmol} / \mathrm{L})$ 2-hr plasma glucose $\geq 200 \mathrm{mg} / \mathrm{dl}(\geq 11.1 \mathrm{mmol} / \mathrm{L})$, history of smoking, serum creatinine level $>1.5 \mathrm{mg} / \mathrm{dl}$. The study subjects were selected among the relatives and attendants of patients, hospital staffs, medical students, subjects available in the Cardiology Department, BSMMU campus, colleagues and also through personal contacts. After selection, objectives and the study procedure were explained to each subject in details and informed written consent were obtained from them. After taking detail personal, medical, family, socioeconomic, occupational and dietary history, physical examination was done and all information were recorded in a data schedule. Height and weight were measured and BMI was calculated. PWV was calculated using following formula. $\mathrm{PWV}=\mathrm{L}$ (m)/TT (s) Here, Length (L) in meter $=$ Carotidofemoral path length. TT(Transit Time) = Td1$\mathrm{Td} 2 . \mathrm{Td} 1=$ The time from peak of $\mathrm{R}$ wave of QRS complex to foot of the femoral pulsed Doppler wave form. $\mathrm{Td} 2=$ The time from peak of $\mathrm{R}$ wave of QRS complex to foot of the carotid pulsed Doppler wave form. (Figure 1)



Figure 1: Assessment of transit time using pulsed Doppler ultrasound after ECG gating.

For calculating TT, pressure wave forms were recorded from the carotid artery and femoral artery using 2D guided pulse wave Doppler. To determine TT, the time from peak of R wave of QRS complex to foot of pulsed Doppler waves were measured by digital calipers. Averages of six heart rate measurements were taken. Carotido-femoral path length in meter was determined by measuring the distances joining from carotid pulse through the supra-sternal notch, the umbilicus and then to femoral pulse by a measuring tape ${ }^{7}$. But usage of superficial

J Bangladesh Soc Physiol. 2018, June; 13(1): 17-21 
distance measurement revealed higher PWV values compared with those obtained using MRI or invasive measures. Hence, a scaling factor of 0.8 derived from Huybrechts et al. were used for converting PWV obtained using superficial distance measurement to 'real' $\mathrm{PWV}^{7}$.

Real PWV $=(0.8 \times$ superficial measurement $) / T T$. Pulse wave velocity measurement was taken in a quiet, temperature controlled room $\left(22 \pm 11^{\circ} \mathrm{C}\right)$. Continuous data were expressed in mean $\pm \mathrm{SD}$. Statistical analysis was done by using independent sample t- test and Pearson correlation coefficient test as applicable. $P$ value $<0.05$ was considered as significant.

\section{Result}

In this study, 17 male and 13 female were included in younger age(19-49 years) group. In older age group, between 50-75 years of age, 19 male and 11 female were studied. Baseline characters of all subjects are presented in Table I. In this study, the PWV was found significantly higher in older age group that of younger age group in both male and female (Figure 2). Moreover, 20\% of older age group subjects had shown abnormally high PWV (Figure 3) whereas, all subjects in younger age group had normal PWV. On correlation analysis, PWV showed significant $(\mathrm{p}<0.001)$ positive correlation with age (Figure 4$)$.



Figure 2: PWV in both age groups of male \& female $(n=60)$



Figure 3: Percentage of abnormally high PWV in older age group healthy subjects.

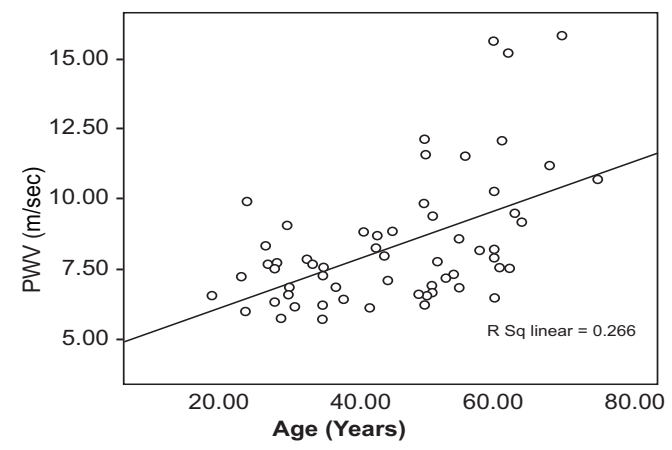

Figure 4: PWV showed significant positive correlation of with age.

Table I: Mean Age, BMI, Pulse \&BP in different groups

\begin{tabular}{lccccc}
\hline Parameters & \multicolumn{2}{c}{ Younger age } & & \multicolumn{2}{c}{ Older age } \\
\cline { 2 - 3 } \cline { 5 - 6 } & Male $(\mathrm{n}=17)$ & Female $(\mathrm{n}=13)$ & & Male $(\mathrm{n}=19)$ & Female $(\mathrm{n}=11)$ \\
\hline Age $($ Years $)$ & $34.64 \pm 7.55$ & $31.46 \pm 6.30$ & & $58.00 \pm 6.21$ & $54.00 \pm 6.19$ \\
BMI $\left(\mathrm{Kg} / \mathrm{m}^{2}\right)$ & $23.64 \pm 1.72$ & $21.92 \pm 2.17$ & & $24.21 \pm 1.54$ & $23.36 \pm .92$ \\
Pulse rate(beats/min) & $71.94 \pm 4.80$ & $70.30 \pm 4.88$ & & $71.15 \pm 4.07$ & $73.09 \pm 4.22$ \\
SBP(mm ofHg) & $123.24 \pm 4.30$ & $124.62 \pm 8.02$ & & $127.63 \pm 6.94$ & $124.09 \pm 12$ \\
DBP(mm ofHg) & $77.35 \pm 5.89$ & $73.07 \pm 5.21$ & & $79.21 \pm 4.49$ & $77.72 \pm 6.06$ \\
\hline
\end{tabular}

Data were expressed as Mean \pm SD. Statistical analysis were done by Independent sample ' $t$ ' test'

J Bangladesh Soc Physiol. 2018, June; 13(1): 17-21 


\section{Discussion}

In the present study, the arterial stiffness was assessed by observing pulse wave velocity in apparently healthy people of different age groups to detect age influence on arterial distensibility.

Higher values of PWV in older age group in both male \& female in this study suggest relative arterial stiffness occurred in older subjects irrespective of gender. This fact was further supported by the significant positive correlation of PWV with age. The present data on presence of abnormal PWV in $20 \%$ subjects of above 50 years of age, indicate some subjects in this age group already developed arterial stiffness. Absence of any abnormal PWV \& comparatively lower value of PWV in younger age group below 50 years provided evidence for their normal arterial elasticity or compliance. Various investigators studied other risk factors for increased PWV leading to increased arterial stiffness.

Lebrun et al found significant positive relationship of PWV with BMI, fasting glucose, diabetes mellitus and triglycerides in an analysis, adjusted for age, mean arterial blood pressure and heart rate. In addition, height and HDL cholesterol were inversely related to PWV. Moreover, they found a linear graded increase in PWV which acts as predisposing risk factor for stroke, coronary heart disease and death. Results of their study on postmenopausal women suggested that increased PWV is a marker for increased risk for stroke, coronary heart disease and death within $10-12$ years $^{8}$. In their study, they used applanation tonometry. In contrast, our study was conducted by doppler method and we excluded CVD, DM, renal failure and used apparently healthy subject to correlate pulse wave velocity with age.

In another study, Alarhabi et al. found that the mean PWV in the group with normal angiography was $8.14 \mathrm{~m} / \mathrm{sec}$ which was significantly different from the patient with coronary artery disease ${ }^{9}$.

Our study was conducted on apparently healthy people who were not affected with hypertension
,DM, renal failure and had no history of smoking .We found normal PWV below age 50 years but PWV gradually increase after 50 years and $20 \%$ subjects developed arterial stiffness . Results of a similar study showed a notable age related increase in arterial stiffness in healthy individuals with no evidence of cardiovascular disease and a low risk factors ${ }^{3}$. They used tonometry for assessing PWV.

Taquet et al. conducted a cross sectional study on PWV in 429 apparently healthy middle aged woman and found weak but significant positive association between PWV and age ${ }^{10}$. The present study showed a strong positive correlation between PWV and age in both male and female.

In a similar study Luiz Pizzi et al. reported PWV were 7.89 and 8.51 in health and disease respectively. It was found that a high degree of correlation of PWV with age progressively increases $6-8 \%$ in each decade of life; this tendency is more pronounced after 50 years. A significant increase of PWV over 50 years was also demonstrated ${ }^{11-12}$ which is similar to this present study.

\section{Conclusion}

From this study, it may be concluded that, arterial stiffness, as measured by the pulse wave velocity by Doppler method, increases linearly with age. Development of arterial stiffness over 50 years of age may be an independent predictor of risk of hypertension.

\section{Conflict of interest: None}

Author affiliations

*1. Amina Begum, Medical Officer, Department of Physiology, Bangabandhu Sheikh Mujib Medical University, Dhaka. Email: dr.aminabegum.bsmmu@ gmail.com

2. Shelina Begum, Professor, Department of Physiology, Bangabandhu Sheikh Mujib Medical University, Dhaka.

3. Jahanara Arzu, Associate Professor, Department of Cardiology, Bangabandhu Sheikh Mujib Medical University, Dhaka.

4. Md. Rezaul Karim Chowdhury, Associate Professor, Department of Hematology, Enam Medical College, Savar, Dhaka.

*For correspondence

J Bangladesh Soc Physiol. 2018, June; 13(1): 17-21 


\section{References}

1. Lee HY and Oh BH. Aging and arterial stiffness. Circ J 2010; 74: 2257-62

2. Salvi P, Safar ME,Carlos L, Borghi C, Patrick L, Athanese B. Heart disease and changes in pulse wave velocity and pulse pressure amplification in the elderly over 80 years: The PARTAGE Study. J Hypertens 2010; 28(10): 2127-33.

3. Gary FM, Helen P, Emelia J, Benjamin MG. Changes in arterial stiffness and wave reflection with advancing age in healthy men and women. Int $\mathrm{J}$ Hypertens 2004; 43:1239-45.

4. Farro I, Fbia D, YaninaZ . Pulse wave velocity as marker of preclinical arterial disease: reference levels in a Uruguayan population considering wave detection algorithms, path lengths, aging and blood pressure. Int J Hypertens 2012;20:882-92.

5. Ranjit R, Binu TG, George V, Madhu KG, Devika P, Subair K,Jayaram V,Mohanan KS, Radhakrishnan VV. Aortic pulse wave velocity and its relationship with complexity of coronary artery disease based on SYNTAX score. Heart Asia 2014; 6:10915. doi: 10.1136/heartasia-2013-010492.

6. Diaz A, Galli C, Matias T, Agustin R. Reference values of pulse wave velocity in healthy people from an urban and rural Argentinean population. $J$ Clin Hypertens 2014; 10:1-7.
7. Razzaque MA, Majumder AAS, Rahman MT, Islam AKMM, Ullah M. Association of aortic pulse wave velocity with the severity of coronary artery disease in patients with acute ST elevation myocardial infarction. Cardiovasc J 2014; 6(2):116-21.

8. Lebrun CEI, van der S, Yvonne T, Bak AAA, Frank $\mathrm{H}$, Pols AP et al. Arterial stiffness in postmenopausal women: determinants of pulse wave velocity. J Hypertens 2002;20(11):2165-72.

9. Alarhabi AY, Mohamed MS, Ibrahim S, Hun TM, Musa KI,Yusof Z . Pulse wave velocity as a marker of severity of coronary artery disease. J Clin Hypertens 2009;11(1):17-21. doi: 10.1111/j.17517176.2008.00061.x

10. Nunez F, Martinez Costa C, Sanchez ZJ, Morata J, Chorro FJ, Brines J .Carotid artery stiffness as an early marker of vascular lesions in children and adolescents with cardiovascular risk factors. Rev Esp Cardiol. 2010;63(11):1253-60. DOI: 10.1016/ S1885-5857(10)70250-4

11. Kohn JC,Lampi MC, Reinhart-King CA. Age-related vascular stiffening: causes and consequences. Front Genet 2015; 6:112.

12. Pizzi OL, Brandäo $\mathrm{AA}$, Pozzan R, Eliane $\mathrm{CM}$, Elizabete VF, Ayrton PB . Pulse Wave Velocity in Young Adults. Study of Rio de Janeiro. Arq Bras Cardiol[internet] 2011;97(1) [cited $6^{\text {th }}$ Aug 2018]:53-58.Available from: Epub May 27, 2011. ISSN 0066-782X. http://dx.doi.org/10.1590/ S0066-782X2011005000067. 